\title{
CRITERIA FOR DETERMINING ELEMENT SIZE AND TIME STEP FOR THERMAL SHOCK SIMULATION
}

\author{
Jinyang Zheng \\ Zhejiang University \\ Yu Quan, Hangzhou, Zhejiang , 310027, China
}

\author{
Rusi P. Taleyarkhan, \& Seokho H. Kim \\ Oak Ridge National Laboratory \\ Oak Ridge, Tennessee, 37831, USA
}

\begin{abstract}
Rapid energy deposition into spallation source targets can lead to their temperature rise at enormous rates, giving rise to dynamic thermoelastic stresses. Understanding and predicting the resulting stress waves are crucial for robust design and safe operation of such devices. To simulate the thermal shock phenomenon accurately, many factors should be carefully considered, such as geometry, surface condition, energy deposition profile, equation of state, possible cavitation, viscous damping, rate-dependent constitutive equation, element size, and time step. In this paper a closed form expression for the induced stress in slender bars with distributed energy deposition has been directly derived; it is then used to test the accuracy of computed results with FEA codes. It was found that significant errors can occur unless care is taken to restrict element size and time step depending on the boundary conditions, steepness of temperature profiles and rise rate. Criteria have been proposed for determining the above two parameters. Numerical simulation with the well-established ANSYS5.5 code system showed that excellent results could be achieved if the proposed criteria are met.
\end{abstract}

\section{NOMENCLATURE}

$c=$ sonic velocity, $c=\sqrt{\frac{E}{\rho}}, \mathrm{m} / \mathrm{s} ;$

$E=$ Young's modulus, $P a$;

$k_{n}=$ eigenvalues, $k_{n}=n \pi / l$;

$l=$ total length of slender bar, $m$;

$l_{o}=$ length of heated portion of slender bar, $m$;

$n=$ integers, $n=1,2,3, \ldots$;

$t=$ time, $s$;

$T(x)=$ temperature rise with respect to reference temperature, ${ }^{\circ} \mathrm{C}$;

$T E=$ total energy of the bar, $\mathrm{J} / \mathrm{m}^{2}$;

$(T E)_{n}=$ total energy associated with the $\mathrm{n}^{\text {th }}$ mode, $\mathrm{J} / \mathrm{m}^{2}$;
$T_{o}=$ temperature, ${ }^{\circ} \mathrm{C}$

$T_{p}=$ period, $s$

$t_{a}=$ finite rise time of temperature, $s$;

$u(x, t)=$ displacement, $m$;

$x=$ coordinate, $m$;

$\alpha=$ coefficient of thermal expansion, $m / m^{\circ} \mathrm{C}$;

$\delta=$ half length of temperature transition, $m$;

$\lambda=$ lame constant;

$\mu=$ lame constant;

$\varepsilon(x, t)=$ strain;

$\rho=$ density, $\mathrm{kg} / \mathrm{m}^{3}$;

$\sigma(x, t)=$ stress, $P a$;

$\sigma^{\prime}(x, t)=$ stress considering the effect of rise-time of temperature, $\mathrm{Pa}$.

\section{INTRODUCTION}

In high energy spallation source systems a significant amount of energy is deposited very rapidly into the target, leading to a rapid temperature rise and stress waves with large amplitudes ${ }^{1-5}$. Understanding and predicting the resulting stress waves due to rapid energy deposition are considered crucial for robust design and safe operation of such devices. Recently, worldwide effort ${ }^{1-5}$ has gone into simulating such thermal shock with various FEA codes, such as CTH, ABAQUS, and ANSYS etc. ${ }^{6-8}$ To simulate such stress waves accurately, many factors should bc carefully considered, such as geometry, surface condition, energy deposition profile, equation of state, possible cavitation, viscous damping, rate-dependent constitutive equation, element size (ES), and time step (TS). It is very difficult and impractical to develop experimental data to investigate the effects of these factors on accuracy of simulation with FEA codes. To determine robust criteria for appropriate $E S$ and $T S$, a simpler method is to use a 
theoretical solution to a relative simple problem and cross-check numerical results.

For reference, in 1974 Sievers $^{9}$ used a step function to simulate the temperature distribution in a cylinder and strived to obtain an analytic solution to the wave equation describing stress wave dynamics in an axially-symmetric cylindrical geometry. In this case, a discontinuity exists at the radial interface region where temperature is rapidly raised and region where temperature remains unchanged. The resulting stress becomes a discontinuous function of time at various times corresponding to the arrival of the infinitely steep-edged wave fronts or their reflections. Thus, the continuous wave equation format cannot apply. Results such as those in Ref.6 are only solutions to a mathematical problem, but the mathematical problem itself ceases to be a correct statement of physical reality. We have determined that a closed-form analytic expression for comparing against FEA codes can be determined in a one-dimensional framework. In this case, derivation of expressions for stress waves in slender bars was found to be most suitable for such a study. However, this is only possible in the absence of step discontinuities. By employing a sinusoidal curve to smooth off the edge of the step temperature distribution function, the authors derived a closed form expression for thermoelastic stress propagation in slender bars; this is then used to judge the accuracy of computed results with FEA codes. The problem geometry is depicted graphically in Figure 1. In subsequent sections we present the derivation of analytic expressions and comparisons against results from a FEA code.

\section{STRESS WAVES IN A SLENDER BAR-PROBLEM FORMULATION}

Energy deposition simulating the stage when highenergy nuclear particles bombard the target was assumed to be spatially distributed as shown in Figure 1. The duration of the particle bursts is of the order of microseconds or even shorter; heat transport is negligible during such short time duration and can be safely neglected in the analytical mode for determining stress waves in the several tens of microseconds time frame.

In the following analysis, a slender bar (a bar being defined as a long rod of circular cross section whose length is at lest 10 times greater than its diameter) of elastic material with no damping, free boundaries, and unchanged material constants is assumed. If, however, the temperature rise is sufficiently high which may cause plastic deformation, more sophisticated methods considering dispersive effects must be adopted ${ }^{10}$. For the present purpose of determining a well-characterized closed-form analytic solution for developing space and time step criteria, neglecting plastic deformation effects is acceptable. We first derive an expression for stress wave propagation for instantaneous temperature rise, and then derive an expression that corresponds to that of the time duration of the nuclear particle pulse (which deposits energy in the target material).

\section{Stress Waves Due to Instantaneous Temperature Rise}

Consider a slender bar of length $l$, whose temperature is instantaneously increased according to the distribution given by Eq.(1) and which remains unchanged, thereafter.

$$
T(x)= \begin{cases}T_{0} & \text { if } 0 \leq \mathrm{x} \leq 1_{\mathrm{o}}-\delta \\ \frac{T_{0}}{2}\left[1-\sin \frac{\pi}{2 \delta}\left(x-l_{o}\right)\right] & \text { if } \mathrm{l}_{\mathrm{o}}-\delta \leq x \leq l_{o}+\delta \\ 0 & \text { if } \mathrm{l}_{\mathrm{o}}+\delta \leq x \leq l\end{cases}
$$

Figure 1 shows the temperature distribution along the bar. Unlike the case of Ref.6 where the temperature gradient is infinite at the heated-unheated interface, in our formulation of Eq.(1) the temperature is differentiable and the half length $\delta$ of the transition interval can become as small as we want to make it. Spatial change in temperature gradient increases as $\delta$ decreases.

The equilibrium equation is now given by

$$
\frac{\partial \sigma(x, t)}{\partial x}=\rho \frac{\partial u(x, t)}{\partial^{2}}
$$

The thermoelastic strain consists of two parts: one component related to stress by Hooke's law and another due to free thermal expansion. The relations is expressed as

$$
\varepsilon(x, t)=\frac{\partial u(x, t)}{\partial x}=\frac{\sigma(x, t)}{E}+\alpha T(x)
$$

Substituting for displacement using Eq.(3) and considering the assumption that the temperature field is independent of time, we can rewrite Eq.(2) as

$$
\frac{\partial^{2} \sigma(x, t)}{\partial x^{2}}=\frac{1}{c^{2}} \frac{\partial^{2} \sigma(x, t)}{\partial^{2}}
$$

Assuming unconstrained boundaries, we obtain

$\sigma(0, t)=\sigma(l, t)=0$

For evaluation of the initial stress and its velocity we assume its initial displacement and velocity are zero. The initial conditions have the form

$\sigma(x, 0)=-E \alpha T(x)$ 
$\frac{\partial \sigma(x, 0)}{\partial}=0$

The solution to Eq.(4), which meets both Eq.(5) and Eq.(7), is well-known ".

$\sigma(x, t)=\sum_{n=1}^{\infty} C_{n} \sin k_{n} \cos k_{n} c t$

From Eq.(8),we get

$\sigma(x, 0)=\sum_{n=1}^{\infty} C_{n} \sin k_{n} x$

Selecting an arbitrary integer $m$, multiplying both sides of the above equation by $\sin \left(k_{m} x\right)$, and integrating from $x=0$ to $l$, and considering the orthogonality of trigonometric series, we obtain,

$C_{n} \int_{0}^{l} \sin ^{2} k_{n} x d x=\int_{0}^{l} \sigma(x, 0) \sin k_{n} x d x$

Remembering that $\sigma(x, 0)$ is given by Eq.(6), we have

$C_{n}=A B_{n}$

where $A=-\frac{2 E \alpha T_{o}}{l}$;

$$
\begin{aligned}
& B_{n}=\frac{1-D_{n}}{k_{n}}-k_{n} \frac{D_{n}}{\frac{\pi^{2}}{4 \delta^{2}}-k_{n}^{2}} ; \\
& D_{n}=\cos \left(k_{n} l_{0}\right) \cos \left(k_{n} \delta\right) .
\end{aligned}
$$

Substituting Eq.(9) into Eq.(8) gives

$$
\sigma(x, t)=A \sum_{n=1}^{\infty} B_{n} \sin k_{n} x \cos k_{n} c t
$$

Displacement is given

$$
u(x, t)=\frac{2 \alpha T_{0}}{l} \sum_{n=1}^{\infty} \frac{B_{n}}{k_{n}} \cos k_{n} x\left(1-\cos k_{n} c t\right)
$$

\section{Stress Waves Due to Finite Temperature Rise-time}

As a matter of fact, an instantaneous rise in temperature cannot occur. For short nuclear particle pulse durations lasting in the nano-to-micro seconds, adiabatic temperature rise occurs, and the rise-time of temperature can safely be assumed equal to the time duration of the pulse itself. Therefore, stress in the bar is given by

$\sigma^{\prime}(x, t)=\int_{0}^{t} \sigma(x, t-\tau) \frac{\partial T(x, \tau)}{T(x) \partial \tau} d \tau$

We assume temperature of the bar is linearly raised, i.e., $\frac{\partial T(x, \tau)}{T(x) \partial \tau}= \begin{cases}\frac{1}{t_{o}} & \text { if } 0 \leq \mathrm{t} \leq \mathrm{t}_{0} \\ 1 & \text { if } \mathrm{t}>\mathrm{t}_{\mathrm{o}}\end{cases}$

Substituting the above equation, and Eq.(10) into Eq.(12) gives

$$
\sigma^{\prime}(x, t)= \begin{cases}\mathrm{A} \sum_{\mathrm{n}=1}^{\infty} \mathrm{B}_{\mathrm{n}} E_{n}(t) \sin k_{n} x & \text { if } 0 \leq \mathrm{t} \leq \mathrm{t}_{\mathrm{o}} \\ \mathrm{A} \sum_{\mathrm{n}=1}^{\infty} \mathrm{B}_{\mathrm{n}} F_{n}(t) \sin k_{n} x & \text { if } \mathrm{t}>\mathrm{t}_{\mathrm{o}}\end{cases}
$$

$$
\begin{aligned}
& \text { where } \begin{aligned}
E_{n}(t) & =\frac{\sin k_{n} c t}{k_{n} c t_{o}} \\
F_{n}(t) & =\frac{\sin k_{n} c t-\sin k_{n} c\left(t-t_{0}\right)}{k_{n} c t_{o}} .
\end{aligned}
\end{aligned}
$$

It can be easily shown that limit of $E_{n}(t), F_{n}(t)$ is $\cos \left(k_{n} c t\right)$ when $t_{o} \rightarrow 0$. This implies that Eqs.(13) reduce to Eq.(10) as time-rise of temperature approaches zero.

\section{SIMULATION WITH FEA CODES}

As is obvious, it is impossible to determine exact solutions to most thermal shock problems. This is due to complex geometrical and multi-material structural considerations. One practical method is to use FEA codes. However, as we point out in this paper, even with stable, convergent solution schemes the accuracy of predictions from FEA codes can be strongly dependent on load step, $E S$ and $T S$. We consider aspects dealing with each of these key parameters as follows.

\section{Load Step Determination}

General wave equation for isotropic linear elastic media is given by Eq.(14) when the body forces except temperature load are neglected ${ }^{12}$.

$(\lambda+\mu) \nabla \nabla \cdot u+\mu \nabla^{2} u-\alpha(3 \lambda+2 \mu) \nabla T=\rho \ddot{u}$

Equation (14) indicates that a thermally induced stress waves are produced by rapid spatial change in temperature. When the wave impinges on a boundary, part of its energy travels forwards while part is reflected back, usually with a phase change. Thereafter the shape of stress at all times is obtained by overlapping of compression and rarefaction component waves.

For the case of continuous spatial change in temperature, the initial stress wavefronts are continuous, whose spatial intervals equal the length of the corresponding temperature transition zone. Both stepped and ramped thermal loads can be appropriately simulated if a small enough $T S$ and fine enough $E S$ are employed. 
However, if spatial temperature jump exists at a boundary or within a restricted domain of the interior, the spatial interval of the initial stress wave front equals $t_{0} c$. where $t_{o}$ is the temperature rise time. When temperature rise time $t_{o}$ is zero, i.e., stepped load, the initial stress is discontinuous at the wave front. Since FEA codes are unable to resolve propagating discontinuities, stepped (i.e., instantaneous) loads cannot be properly simulated.

In an accelerator facility target, energy is rapidly deposited by proton beam through a so-called window region. The situation is similar to that shown in figure 1 for a slender bar if we imagine that a proton beam enters the bar from the left and deposits its energy to provide a temperature rise distribution as shown therein. In this case, at the left-most boundary region to the right of the boundary (i.e., in the $0+$ direction of the $x$-axis) the temperature distribution is continuous and differentiable. However, since the left boundary is free to move, the temperature distribution left of the origin (in the 0direction of the $\mathrm{x}$-axis) is discontinuous and nondifferentiable. Therefore, for FEA simulation purposes if a stepped load is applied as a convenience feature as in Ref. 1 or 3, a spatial temperature jump will exist at its front (left boundary) face. This discontinuity cannot be appropriately simulated since the time-and-spatial step requirements become identically equal to zero.

\section{Element Size Determination}

As mentioned above, properly resolving spatial temperature differentials in the region where large temperature gradients exist plays an important role. As may be obvious, when spatial temperature changes become increasingly rapid, accurate numerical representation requires increasingly finer meshing. We shall take the temperature described by Eq.(1) as an example to study how small the mesh should be for accurate simulation.

Let $\triangle$ stand for $E S$ in the vicinity of rapid temperature transition and $\delta / \Delta=N$. The temperature gradient is calculated in FEA codes in accordance with the following equation.

$\frac{D T(x)}{D x}=\frac{T(x+\Delta, t)-T(x, t)}{\Delta}$

Inserting temperature at the transition region given by Eq.(1) into the above equation, we get

$\frac{D \pi(x)}{D x}=-\frac{T_{0} \pi}{4 \delta} \cos \frac{\pi}{2 \delta}\left(x-l_{o}+\frac{\delta}{2 N}\right) \frac{\sin \frac{\pi}{4 N}}{\frac{\pi}{4 N}}$
The limit of $\frac{D \pi(x)}{D x}$ when $N$ approaches infinite can be obtained by applying L'Hopital's rule,

$\operatorname{limit}_{x \rightarrow \infty} \frac{D T(x)}{D x}=-\frac{T \pi}{4 \delta} \cos \frac{\pi}{2 \delta}\left(x-l_{o}\right)=\frac{d T(x)}{d x}$

where $d T(x) / d x$ is the exact temperature gradient. The above equation means that the accuracy of simulation depends strongly on $N$, i.e., the larger the $N$, the better the prediction. But, an $N$ that is too large may improve accuracy only slightly and results in a calculation that is prohibitively expensive. A tradeoff will obviously need to be made by the numerical experimentalist. For example, when $N$ equals 8 or $80, \sin (\pi / 4 N) /(\pi / 4 N)$ is 0.998 and 0.9998, respectively. Numeric calculations and comparisons we have made show that temperature gradient can be appropriately estimated by Eq.(15) when $N$ is not less than 8 . To adequately resolve the spatial distribution of temperature, the following criterion is recommended to determine ES.

$\Delta \leq \frac{\delta}{8}$

For a multi-dimensional simulation, If the temperature varies gradually similar to the sinusoidal function assumed herein for the case of a single coordinate system, the value for ES given by Eq.(16) should be able to resolve steep temperature gradients all around. Therefore, Eq.(16) is expected to be applicable to higher- dimension situations also.

\section{Time Step Determination}

Equation (11) reveals that the displacement function consists of various modes. We derived the condition for time step determination via consideration of the most dominant frequency impacting the energy of the system. We first started by obtaining the sum (TE) of the instantaneous kinetic- and potential-energy of the slender bar by applying Eq.(10) and (11) as

$$
T E=\frac{E \alpha^{2} T_{0}^{2}}{l} \sum_{n=1}^{\infty} B_{n}^{2}
$$

The total energy associated with the nth mode is given by

$$
(T E)_{n}=\frac{E \alpha^{2} T_{0}^{2}}{l}\left\{\frac{1-D_{n}}{k_{n}}+D_{n}\left[\frac{1}{2\left(\frac{\pi}{2 \delta}+k_{n}\right)}-\frac{1}{2\left(\frac{\pi}{2 \delta}-k_{n}\right)}\right]\right\}^{2}
$$


Inspection of this result reveals that the most important modes are those which minimize the denominator $(\pi / 2 \delta$ $k_{n}$ ), i.e., those for which $k_{n} \sim \pi / 2 \delta$. When $k_{n}$ approaches $\pi / 2 \delta$, to simplify the analysis we can neglect the first two terms in the parenthesis and write

$(T E)_{n}=\frac{E \alpha^{2} T_{0}^{2}}{4 l} \cos ^{2} k_{n} l \frac{\cos ^{2} k_{n} \delta}{\left(\frac{\pi}{2 \delta}-k_{n}\right)^{2}}$

Let $\frac{\pi}{2 \delta}=\frac{\Omega}{c}$, and $\beta=\frac{\Omega-\omega_{n}}{\mathrm{c}}$. Therefore, the above equation can be written as

$(T E)_{n}=\frac{E \alpha^{2} T_{0}^{2} \delta^{2}}{4 l} \cos ^{2}\left(\frac{\omega_{n} l_{o}}{c}\right) \frac{\sin ^{2} \beta \delta}{(\beta \delta)^{2}}$

Since circular frequencies are discrete, the $(T E)_{n}$ vs. $\omega_{\mathrm{n}}$ described by the above equation can be obtained by equally dividing the abscissa under the envelope curve to obtain.

$T E(\omega)=\frac{E \alpha^{2} T_{o}^{2} \delta^{2}}{4 l} \cos ^{2} \frac{\omega_{o}}{c} \frac{\sin ^{2}\left[\delta\left(\frac{\Omega-\omega}{c}\right)\right]}{\left[\delta\left(\frac{\Omega-\omega}{c}\right)\right]^{2}}$

The above equation shows that the circular frequencies that have the most important energy contribution are those for which $\left|\delta \frac{\Omega-\omega}{c}\right| \leq \pi$. Hence the maximum important circular frequency is $3 \pi c / 2 \delta$. To resolve this frequency, time step should be determined by

$T S \leq \frac{1}{20} \frac{2 \pi}{\omega_{\max }}=\frac{\delta}{15 c}$

In the case of finite rise-time of temperature, the corresponding length of temperature transition zone is $t_{o} c$. To resolve this, the smaller one of $\delta$ and $t_{o} c / 2$ should be used when determining $E S$ and $T S$.

In the case of finite rise-time of temperature, the corresponding length of temperature transition zone is $t_{0} c$. To resolve this, the smaller one of $\delta$ and $t_{o} c / 2$ should be used when determining $E S$ and $T S$.

Integration methods used in FEA codes fall into two categories: explicit and implicit. It has been found that ${ }^{13}$, for wave-propagation problems, the time step for implicit methods must be about the same as that for explicit methods to satisfy accuracy requirement.

So far we found from the open literature that no rigorous stability criterion for determining proper time step has been developed, but it is customary to determine the $T S$ from $^{13}$ as

$T S=(06 \sim 0.9) \frac{\Delta}{c}$

The time step size predicted by Eq.(17) which is based on theoretical analysis clearly meets and is more limiting than the above (sound-speed limiting) and experience based requirement. Therefore, Eq.(17) may be used to determine $T S$ for stable computation of thermal shock conditions.

\section{COMPARISONS OF ANALYTIC SOLUTIONS AND FEA PREDICTIONS}

The well-established ANSYS5.5 code was employed for comparison of FEA predictions against exact analytic solutions presented in this paper. This was done to calculate the thermally-induced stresses in a slender bar (one dimensional case shown in Figure 1), and for a cylindrical bar (two dimensional case where temperature jump conditions are absent at boundaries) subjected to rapid energy deposition.

\section{One Dimension Problem}

Stresses in a tungsten slender bar subjected to the temperature distribution described by Eq.(1) and shown graphically in Figure 1, were simulated using ANSYS5.5 for comparison against analytic results. Clearly, there is one temperature jump at the left boundary of the bar, and a temperature transition zone at the heated and unheated interface. Due to this configuration, two stress waves, $a$ and $b$, propagating in opposite direction are produced at the temperature transition zone. At the left end a stress wave $c$ is generated. When these stress waves reach one of the ends of the bar, they will be reflected with a change in phase. The motion dynamics of wave transport is depicted in Figure 2 that represents the wave characteristics for the slender bar case. With such a depiction, the stress at any point can be determined by superposition of the three fundamental stress waves.

As mentioned previously, a temperature jump (discontinuity) condition exists at the left end of the bar and as such, cannot be simulated with the ANSYS 5.5 FEA code. Only a ramped (temperature) load can be simulated since ANSYS 5.5 (like other FEA code) does not incorporate propagation of discontinuous wave front. Three cases with different temperature rise time were calculated. In order to check the consequences of forcing ANSYS 5.5 to simulate a stepped temperature load in time (i.e., simulation of results with zero temperature rise time). A stepped load was applied in case 4. Pertinent parameters used in the calculations are as follows: 
$l=6 \mathrm{~mm}, l_{\mathrm{o}}=2.5 \mathrm{~mm}, E=4.43 \times 10^{11} \mathrm{~Pa}, \alpha=7.936 \times 10^{-6}$ $\mathrm{m} / \mathrm{m}^{\circ} \mathrm{C}, c=4791 \mathrm{~m} / \mathrm{s}, \rho=19300 \mathrm{~kg} / \mathrm{m}^{3}, \delta=0.8 \mathrm{~mm}, E S=$ $10 \mu \mathrm{m}, T S=2 n s$. ES and TS required by criteria Eq.(16) and (17) were given in table 1 . It can be seen that the criteria are met in case 1, but are not obeyed in other three cases. Calculated stress and the exact result given by Eq.(13) at the point $x=4 \mathrm{~mm}$ are summarized in Table 2.

As is clear from table 2, the stress magnitude, frequency, and shape are in excellent agreement when the proposed criteria are met. In case 1 the relative error between prediction and exact result is about $8 \%$, which is usually acceptable in engineering calculation. For improved accuracy the criteria of Eq.(16) and (17) need to be made stricter. In the other three cases, ES and TS required at the left end are not met, i.e., the wave front of the initial stress wave $c$ is not properly simulated. Rapid stress oscillations occur at the corresponding time when stress wave $c$ arrives at that point. This gives rise to very significant discrepancies and clearly indicates the importance of restricting element size and time step.

\section{Two Dimensional Problem}

To enable determination of appropriateness of use of the criteria suggested by Eqs.(16) and (17) for multidimensional geometries, a two dimensional case was considered (even though we do not have an exact solution to compare against now). Numerical simulations were made to predict stress waves in a long cylinder heated rapidly along its axis with constant temperature in the axial direction and radial-varying spatial temperature distribution. Radial temperature distribution was kept similar to that described by Eq.(1) with $r$ and $r_{v}$ taking the place of $x$ and $l_{o}$.Fixed outer boundaries and zero axial displacement were assumed. There is only one temperature transition zone at the heated-unheated interface. It is to be noted that, no jump exists in the spatial temperature distribution, even at the outer boundary since it is fixed. As a consequence, a stepped load in time may be applied. The cylinder radius $R$ is $5 \mathrm{~mm}$; radius of heated region $r_{o}=2.5 \mathrm{~mm}$; half length of temperature transition $\delta=1 \mathrm{~mm}$; temperature $T_{o}=890^{\circ} \mathrm{C}$.; temperature rise time $t_{o}=0$.

$E S$ and TS in accordance with Eq.(16) and (17) criteria are $0.125 \mathrm{~mm}$ and $12.5 \mathrm{~ns}$ respectively. Four cases were calculated: case 1, $E S=0.01 \mathrm{~mm}, T S=12.5 \mathrm{~ns}$; case 2, $E S=0.1 \mathrm{~mm}, T S=12.5 \mathrm{~ns}$; case $3, E S=1 \mathrm{~mm}, T S=40 \mathrm{~ns}$; and case $4, E S=1 \mathrm{~mm}, T S=1 \mathrm{~ns}$. Obviously, case 1 and case 2 meet the proposed criteria, while case 3 and 4 do not comply with the proposed criteria.

As expected, no stress attenuation was noted for case 1. Since $E S$ in case 2 is larger than that in case 1 , slight stress attenuation occurs. However, the difference in the stress amplitudes is small. In case 3 and 4 , not only significant stress reduction are predicted, but also the amplitudes of stresses were noted to be much smaller than that in case 1 . This further goes to indicate the importance of paying attention to the importance of adequately choosing $E S$ and $T S$ for thermal shock simulation, as otherwise, totally misleading and physically unrealistic results can be predicted with FEA codes.

\section{CONCLUSIONS/SUMMARY STATEMENTS}

1. Exact thermoelastic stress solutions for a onedimensional geometry slender bar with spatiallyvarying, time dependent energy deposition have been directly derived and used to test the accuracy of FEA code predictions.

2. Significant discrepancies can occur unless care is taken to restrict both the ES and TS. The stress magnitude, frequency, and shape can be reasonablywell (within 10\%) predicted when the proposed criteria of Eq.(16) and (17) are met in the simulation model with FEA.

3. Stepped thermal load should not be applied in FEA simulations for thermal shock of SNS-type target if FEA codes are not designed to resolve propagation of discontinuous wave fronts.

4. Although we present comparisons for only the cases of one-dimensional and two dimensional waves, the criteria developed are quite general and may be used for generalized multidimensional thermal shock simulation of SNS target systems.

\section{REFERENCES}

1. Futakawa, M., etc., 2000,"Pressure and Stress Waves in a Spallation Neutron Source Mercury Target Generated by High-power Proton Pulses," Nuclear Instruments and Methods in Physics Research A, Vol.439,pp:1 7

2. Kim, S.H.,etc., 1999," Thermal Shock Analysis of the BNL/AGS Mercury Target Experiments," Transactions American Nuclear Society, Vol.80,pp: 312 313

3. Ni, L. and Bauer, G.S.,1998, "Dynamic Stress of a Liquid Metal Target Container Under Pulsed Heating," ASME Journal of pressure Vessel Technology, Vol.120, pp:359 364

4. Taleyarkhan, R.P.,ctc., 1997, "Thermal Shock Assessments for National Spallation Neutron source target System," Proceedings of 1997 International Meeting on Advanced Reactors safety, Florida

5. Taleyarkhan, R.P., etc.,1999,"Modeling \& Analysis of AGS(1998) Thermal Shock Experiments," Proceeding of the Third International Topical Meeting on Nuclear Application of Accelerator Technology, Long Beach,CA. 
6. Mcglaun, J.,etal., 1990, "CTH: A Three Dimensional Shock Wave Physics," Int. J. Impact Eng.

7. ANSYS ,1999, Revision 5.5, Swanson Analysis System,Inc.

8. ABAQUS/Explicit, Version 5.6, Hibbitt Karlesson Sorensen,Inc

9. Sievers, P., 1974, "Elastic Stress Waves in Matter Due to Rapid Heating By an Intense High-energy Particle Beam," European Organization for Nuclear Research, Germany
10. Beltzer, A.I.,1988, "Acoustics of Solids," Berlin: Springer Verlag, pp:125 173

11. Dudley, H. Towne, 1967, "Wave Phenomena," London: Addison Wesley Publishing Company, pp:322 371

12. Achenbach, J.D.,1993,"Wave Propagation in Elastic Solids," London, North-holand Publishing Company,pp:391 392

13. Jonasa A.Zukas, etc.,1982, "Impact Dynamics," New York: John Wiley \&Sons,pp:379 382

Table 1. ES and TS Required By Eq.(16) and (17)

\begin{tabular}{|c|c|c|c|c|c|c|}
\hline $\begin{array}{c}\text { Case } \\
\text { No. }\end{array}$ & $\begin{array}{c}\text { Temperature } \\
\text { Rise Time, ns }\end{array}$ & \multicolumn{2}{|c|}{$\begin{array}{c}E S \text { and } T S \text { required at } \\
\text { Temperature Transition Zone }\end{array}$} & \multicolumn{2}{|c|}{$\begin{array}{c}E S \text { and TS required at } \\
\text { Left end }\end{array}$} & $\begin{array}{c}\text { Whether } E S \text { and } \\
\text { TS Meet Eq.(16) } \\
\text { and (17) }\end{array}$ \\
\cline { 3 - 6 } & & ES, $\mu \mathrm{m}$ & TS, ns & ES, $\mu \mathrm{m}$ & TS,ns & yes \\
\hline 1 & 60 & 100 & 11.1 & 17.9 & 12.0 & no \\
\hline 2 & 30 & 100 & 11.1 & 9.0 & 1.0 & no \\
\hline 3 & 10 & 100 & 11.1 & 3.0 & 0.33 & no \\
\hline 4 & 0 & 100 & 11.1 & 0 & 0 & 0 \\
\hline
\end{tabular}

Table 2. Comparison Between Calculated Stress and Exact Result

\begin{tabular}{|c|c|c|c|c|c|c|}
\hline Case No. & \multicolumn{3}{|c|}{ Maximum Compression Stress } & \multicolumn{3}{c|}{ Maximum Tensile Stress } \\
\cline { 2 - 7 } & $\begin{array}{c}\text { Calculated } \\
(\mathrm{GPa})\end{array}$ & $\begin{array}{c}\text { Exact } \\
(\mathrm{GPa})\end{array}$ & $\begin{array}{c}\text { Relative } \\
\text { Error(\%) }\end{array}$ & $\begin{array}{c}\text { Calculated } \\
(\mathrm{GPa})\end{array}$ & $\begin{array}{c}\text { Exact } \\
(\mathrm{GPa})\end{array}$ & $\begin{array}{c}\text { Relative } \\
\text { Error(\%) }\end{array}$ \\
\hline 1 & -1.69 & -1.57 & -7.64 & 2.84 & 2.84 & 0 \\
\hline 2 & -1.81 & -1.57 & -15.2 & 2.86 & 2.84 & 0.7 \\
\hline 3 & -2.11 & -1.57 & -34.4 & 2.86 & 2.84 & 0.7 \\
\hline 4 & -2.15 & $\mathrm{NA}$ & & 2.86 & NA & \\
\hline
\end{tabular}




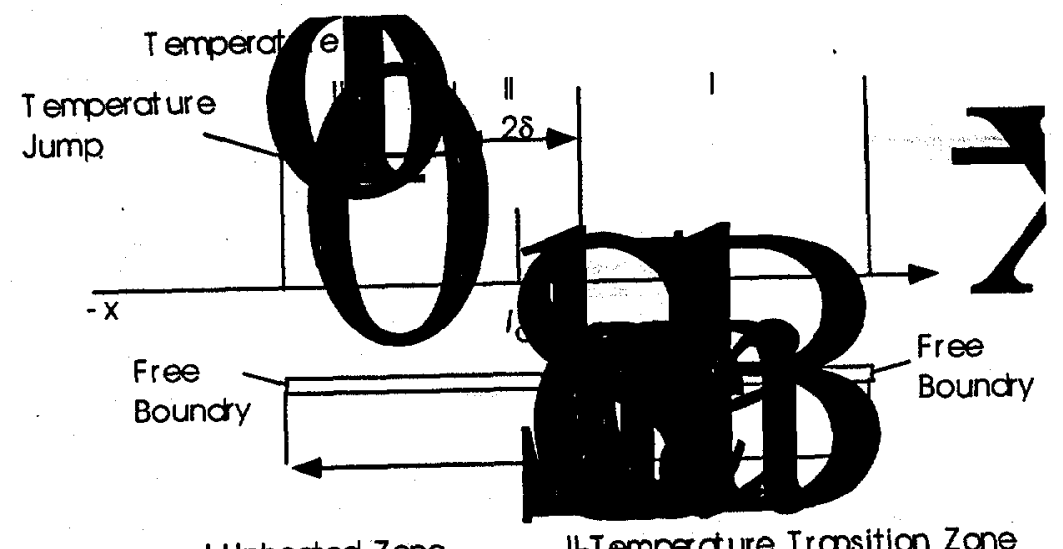

1-Unheded Zone

ItTemperdure Transition Zone

III-Hected Zone With Even Temperature Distribution

Figure 1. Assumed temperature distribution for slender ber

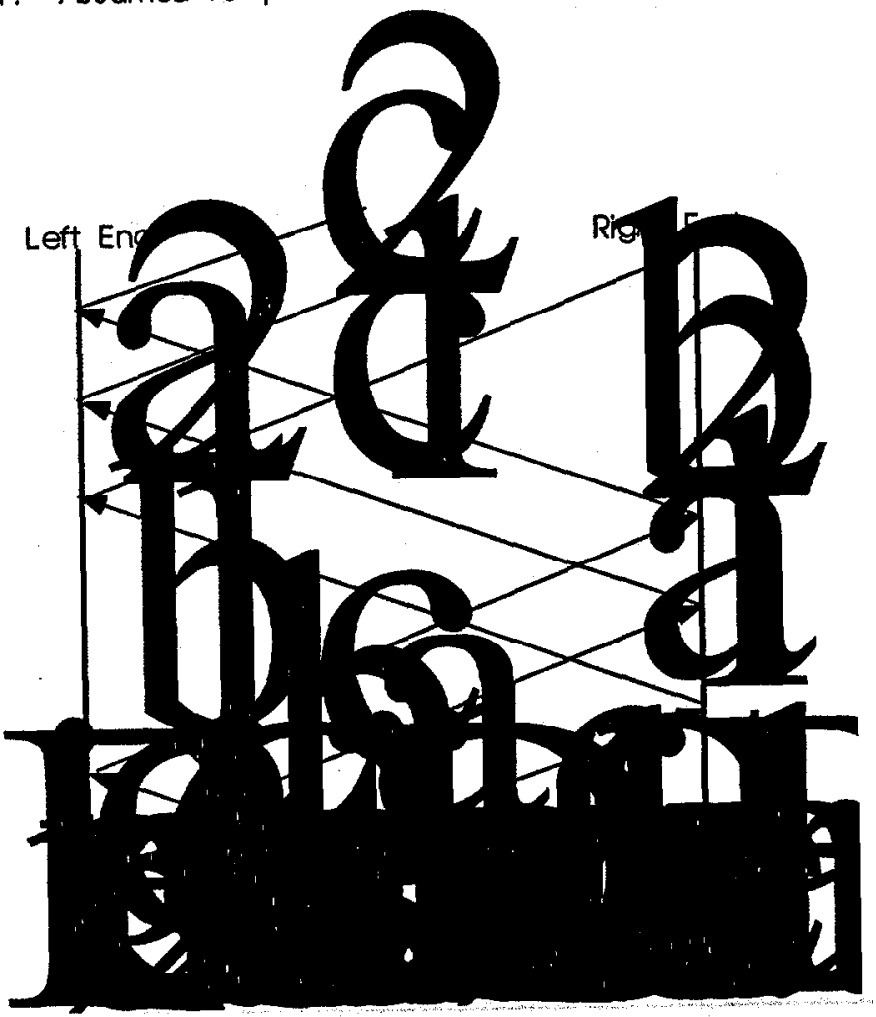

